\section{Chalazia development in multiple myeloma: a new complication associated with bortezomib therapy}

\author{
Charles Yun, ${ }^{1}$ Nikhil Mukhi, ${ }^{1}$ \\ Valerie Kremer, ${ }^{1}$ Roman Shinder, ${ }^{2}$ \\ Vaibhav Verma, ${ }^{1}$ Olcay Batuman ${ }^{1}$ \\ 1Division of Hematology/Oncology, \\ 2Department of Ophthalmology, SUNY \\ Downstate Medical Center, New York, NY, \\ USA
}

\begin{abstract}
Multiple myeloma (MM) is a neoplasm of plasma cells within the bone marrow. A major impact on improving survival in MM has been the use of the boronic acid-derived proteasome inhibitor bortezomib, a first-in-class selective inhibitor of the $26 \mathrm{~S}$ proteasome. Ocular side effects of bortezomib are rare. In this report, we present 2 patients with active MM in whom persistent chalazia became a therapy-interfering complication of treatment with bortezomib. Both patients had relapsed ISS III B kappa light chain myeloma, and they were responding to treatment with bortezomib until chalazia - which caused intolerable discomfort - started. In both patients discontinuation of bortezomib was necessary for chalazia to heal, and restarting of bortezomib was associated with relapse of chalazia.
\end{abstract}

\section{Introduction}

Multiple myeloma (MM) is a neoplasm of plasma cells within the bone marrow. Despite the significant improvements in patient outcomes as a result of drug therapies with immunomodulators, high-dose chemotherapy (HDCT) with stem cell rescue (SCT) and proteasome inhibitors, resistance to treatment ultimately develops and MM remains incurable. ${ }^{1}$ A major impact on improving survival in MM has been the use of the boronic acidderived proteasome inhibitor bortezomib, a first-in-class selective inhibitor of the $26 \mathrm{~S}$ proteasome. ${ }^{2}$ Wide usage of bortezomib in patients with MM based on its potent antimyeloma effects even in prognostically challenged MM patients resulted in better understanding of the side effects of this drug. The most frequent (in $>30 \%$ of patients) of these drug-associated toxicities include: fatigue, generalized weakness; peripheral neuropathy characterized by decreased sensation and paresthesia in hands and feet, nausea and vomiting, diarrhea, poor appetite, constipation, anemia and thrombocytopenia. ${ }^{3}$ Unfortunately, some of these effects interrupt with treatment schedule, dose reduction, or discontinuation even in the absence of drug resistance. It is therefore important to understand and treat potentially new side effects in order to be able to deliver best advantage that bortezomib can provide in drug-sensitive patients with MM.

Ocular side effects of bortezomib are rare. In this report we present 2 patients with active MM in whom persistent chalazia became a therapy-interfering complication of treatment with bortezomib. Both patients had relapsed ISS III B kappa ( $\kappa)$ light chain myeloma, both patients were responding to treatment with bortezomib until chalazia which caused intolerable discomfort, started. In both patients discontinuation of bortezomib was necessary for chalazia to heal, and restarting of bortezomib was associated with relapse of chalazia.

\section{Case Report \#1}

A 56 year old African-American female presented in 2006 with a 2 month history of worsening pain in rib cage and upper back. Physical exam was positive for point tenderness on multiple ribs and in thoracic spine. Complete blood count displayed: white blood count (WBC) $2990 / \mathrm{mm}^{3}$ with normal differential, hemoglobin (HB) $12.5 \mathrm{~g} / \mathrm{dL}$, platelet count $144,000 / \mathrm{mm}^{3}$; blood urea nitrogen (BUN) $50 \mathrm{~g} / \mathrm{dL}$, serum creatinine (Cr) 5.6 $\mathrm{mg} / \mathrm{dL}$, calcium $10.2 \mathrm{mg} / \mathrm{dL}$, b2 microglobulin $16.5 \mathrm{mg} / \mathrm{L}$ and albumin $3.0 \mathrm{mg} / \mathrm{dL}$. Liver function tests and serum protein electrophoresis were within normal limits. 24 hours urine protein electrophoresis displayed $2 \mathrm{~g} / \mathrm{L}$ bband. Skeletal X-ray survey showed disseminated lytic lesions. Bone marrow biopsy showed 6\% monotypic plasma cells expressing $\kappa$ light chains with normal cytogenetics. She was diagnosed with ISS Stage IIIB $\kappa$ light chain myeloma was treated with upfront thalidomide $200 \mathrm{mg}$ per os/day, dexamethasone $40 \mathrm{mg}$ per os /week and Doxil $20 \mathrm{mg} / \mathrm{m}^{2}$ intravenously, with the intention to receive HDCT and ASCT. After 4 cycles (120 days) she achieved a very good partial remission (VGPR) with disappearance of the $\kappa$-light chains in the urine by immunofixation and resolution of $50 \%$ of the skeletal lytic lesions. She refused further therapy. However, returned to clinic on January of 2014 with a painful new rib lytic lesion detected on X-ray. Serum free $\kappa$ light chains were $3020 \mathrm{mg} / \mathrm{L}$ and free $\lambda$ light chains were $8.30 \mathrm{mg} / \mathrm{L}$ with a $\kappa / \lambda$ ratio of 364 . Bortezomib $1.3 \mathrm{mg} / \mathrm{m}^{2} \mathrm{sc}$ and dexamethasone $40 \mathrm{mg}$ po weekly was started. After 2 cycles (36 days) she developed bilater-
Correspondence: Nikhil Mukhi, SUNY Downstate Medical Center, 450 Clarkson Avenue, Brooklyn, 11203 Box 20, New York City, NY, USA.

Tel.: +1.718.270.1500 - Fax: +1.718.270.1578.

E-mail: drnikhilmukhi@gmail.com

Key words: Chalazion; eye disease; bortezomib; multiple myeloma.

Contributions: the authors contributed equally.

Conflict of interest: the authors declare no potential conflict of interest.

Received for publication: 23 November 2014.

Revision received: 8 March 2015

Accepted for publication: 16 April 2015.

This work is licensed under a Creative Commons Attribution NonCommercial 3.0 License (CC BYNC 3.0).

CC Copyright C. Yun et al., 2015

Licensee PAGEPress, Italy

Hematology Reports 2015; 7:5729

doi:10.4081/hr.2015.5729

al subcutaneous mildly tender, extremely itchy upper eyelid lesions $7 \times 10 \mathrm{~mm}$ that were diagnosed by ophthalmology as acute chalazia (Figure 1A,B) with coexisting mild conjunctivitis. Bortezomib was held, and the eye the lesions were managed conservatively with warm saline compresses applied TID at home by the patient. Bortezomib was restarted after a 2 week lapse, as she had symptomatic relief from ophthalmic treatment, chalazia decreased in size by $>75 \%$ and conjunctivitis cleared. After the second week of bortezomib reinitiation additional multiple bilateral chalazia with painful conjunctivitis developed, at which point bortezomib was held another week and she ophthalmology prescribed topical tobramycin/dexamethasone eye drops applied TID during the day and in ophthalmic ointment form to be applied at night, along with continued use of warm compresses. This treatment led to improvement of her eye lesions while on bortezomib (Figure 1C). Patient currently continues to receive bortezomib $1.3 \mathrm{mg} / \mathrm{m}^{2}$ weekly with dexamethasone $20 \mathrm{mg} /$ week and uses topical tobramycin/dexamethasone eye drops. Her most recent serum free $\kappa$ light chains are $2284 \mathrm{mg} / \mathrm{L}$ and free lambda $(\lambda)$ light chains are $13.20 \mathrm{mg} / \mathrm{L}$ with a $\kappa / \lambda$ ratio of 173 . Bone marrow showed $30 \%$ cellularity with $10-15 \%$ K-restricted plasma cells. FISH showed gain of 1q21; monosomy 13; gain of 9,15 and gain of FGFR3/4p, CCND1/11q, IGH14q sequences; and loss of MAF/16q, indicating that biology of her disease had changed for the worse since 2006 . She is willing to have her stem cells collected for possible ASCT/HDCT. 


\section{Case Report \#2}

A 70-year-old African-American male presented in August, 2013 with left shoulder pain and inability to move his left arm. On physical exam, he had severe tenderness on palpation at the humoral shaft, and was unable to perform any movement. There were no other findings. Complete blood count displayed WBC $6200 / \mathrm{mm}^{3}$ and normal differential, HB $14.9 \mathrm{~g} / \mathrm{dL}$, platelet

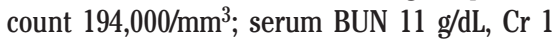
$\mathrm{mg} / \mathrm{dL}$, b-2 microglobulin $3.5 \mathrm{mg} / \mathrm{L}$, Ca 9.2 $\mathrm{mg} / \mathrm{dL}$. Liver function tests and serum protein electrophoresis were normal. Serum free $\kappa$ light chains were $1430 \mathrm{mg} / \mathrm{L}$ and free $\lambda$ light chains were $8 \mathrm{mg} / \mathrm{L}$ with a $\kappa / \lambda$ ratio of 175 . Skeletal Xray survey revealed diffuse lytic lesions and a pathological fracture of the left humeral shaft. Bone marrow biopsy showed 20\% atypical plasma cells expressing $\kappa$-light chains with normal cytogenetics. He underwent bilateral femoral shaft and right humerus fixation surgeries, followed by palliative radiotherapy to bilateral shoulders and femurs.

Patient was next started on systemic treatment with lenalidomide $25 \mathrm{mg}$ per os daily Day 1-21, dexamethasone 40 mg Day 1, 8, 15, 22 (Q 28 days) with zoledronic acid $4 \mathrm{mg}$ IV monthly. He had partial remission (PR) to treatment after 2 cycles as evidenced by persistence of free light chains in the urine. He was switched to bortezomib $1.3 \mathrm{mg} / \mathrm{m}^{2} \mathrm{SC}$, cyclophosphamide $300 \mathrm{mg} / \mathrm{m}^{2}$ and dexamethasone 40 mg per os weekly (VCD). The serum $\kappa / \lambda$ quantitative light chains and ratio normalized after 4 cycles. After second cycle of VCD, he developed redness and swelling of both eyelids with a single nodule with central crusting, this was diagnosed as a chalazion by ophthalmology. He was initially treated with warm saline compresses but his symptoms did not improve with conservative management. He subsequently required incision and drainage of one of the lesions, with tobramycin/dexamethasone eye drops and oral doxycycline treatment leading to resolution of the chalazion over 1 month. He was continued on the bortezomib based regimen and after the fourth cycle, however, he developed multiple bilateral chalazia leading to significant treatment delay. Patient elected to stop treatment after 6 cycles due to Grade III diarrhea on bortezomib. At six week post discontinuation follow up, patient's chalazia resolved. He is currently being observed off treatment.

\section{Discussion and Conclusions}

Bortezomib's antimyeloma effect is largely due to inhibition of endoplasmic reticulumdependent degradation (ERAD) of excessively synthesized abnormal (myeloma) M proteins, which provoke ER stress and cause apoptosis. ${ }^{4}$

When used as a single agent, and especially in combination with other antimyeloma agents, bortezomib is robustly effective, and is FDA approved in the treatment of upfront, relapsed and refractory MM, and in patients who have previously responded well to bortezomib. ${ }^{1}$ Despite its wide usage, there have been few reports of ocular side effects of bortezomib. ${ }^{5}$

Chalazion or meibomian cyst is a benign lipogranulomatous collection which arises from one of the meibomian glands lining the tarsal plate of the eyelid. ${ }^{6}$ It is caused by lipid inspissation in the meibomian glands, which ruptures and releases lipid from the gland into the surrounding tissues. This leads to a granulomatous inflammatory reaction. 7,8

As described in the two cases presented here, patients present with eyelid edema, tenderness and a lump on the eyelid developing over weeks. Larger lesions can cause mechanical ptosis. ${ }^{8}$ About $46 \%$ of chalazia resolve with conservation management with hot compresses, but in refractory cases, topical or intralesional steroids, oral doxycycline and surgical excision, have been used. ${ }^{9}$

This case report sheds light on the associa-

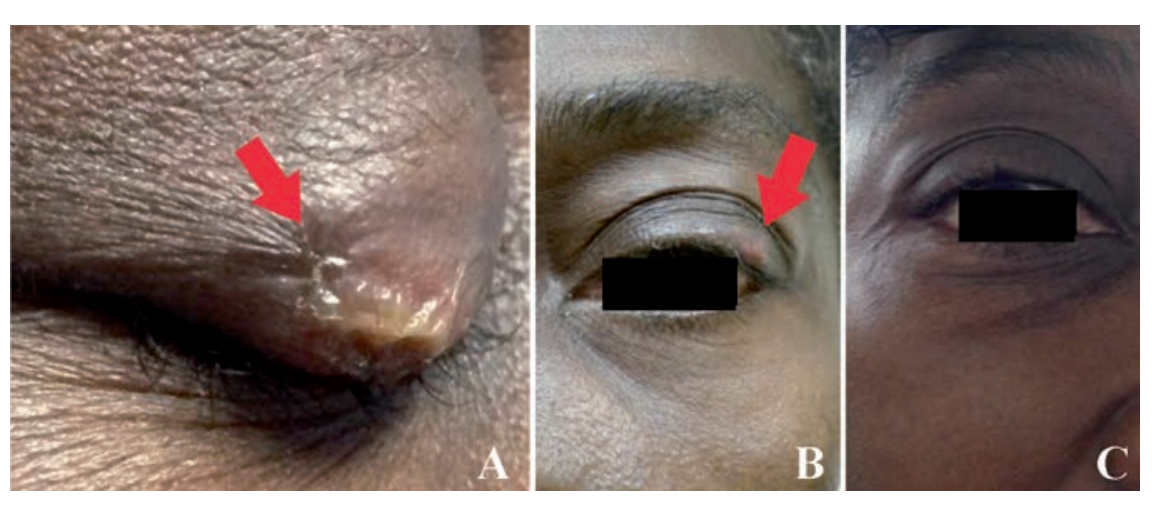

Figure 1. Patients' upper eyelid lesions (A, B) and their improvement (C) tion between bortezomib with chalazia. Since the approval of bortezomib for MM in 2003, it has now become an important backbone of major treatment regimens in frontline as well as relapsed MM patients who were refractory or nonrefractory. ${ }^{1}$ The addition of bortezomib has contributed significantly to the survival of MM patients at all prognostic stages of their disease. It is important to monitor and detect potential side effects so as to maximize the benefit by avoiding side effects that lead to dose reduction or discontinuation and allow patients to have full benefits of this agent.

Chalazion is a lesser known side effect of this drug which may be due to under-reporting. There are currently 2 studies that report chalazion association with bortezomib therapy. One phase II study of bortezomib with rituximab in relapsed refractory Waldenstrom's macroglobulinemia reported 6 of 37 (16\%) patients with conjunctivitis/chalazia although no distinction was made between the two. ${ }^{5}$ Grob SR et al. reported a case series of 6 patients who developed chalazia while on bortezomib therapy with a mean 3.3 months after exposure (range 1-6 months). ${ }^{10}$ All patients had failed conservative measures including warm compresses and lid hygiene. Four patients underwent incision and curettage and 5 of 6 patients had bortezomib suspended or discontinued owing to eye discomfort in the case series. ${ }^{10}$ In our patients the mean time to development of chalazia after start of bortezomib was 30 days. Both patients we report had $\kappa$-light chain myeloma. It is unclear if this is a coincidental finding or if this side effect is more common or exclusive to light chain or specifically $\kappa$-light chain MM. It led to treatment delay in both our patients and one patient required surgical intervention.

We therefore recommend careful eye exam, an ophthalmology consultation as symptoms or signs of itching or redness present. Early detection and rapid treatment with topical corticosteroid eye drops during the day and in ointment form at night in addition to excellent eye hygiene by conventional warm compress and by frequent hand washing is a reasonable approach in order to minimize treatment delay. In those patients in whom chalazia persist or relapse after the preceding therapy it may be prudent to either switch to other proteasome inhibitors or consider surgical intervention for the chalazia.

Other drugs have also been implicated in chalazia development. The use of topical prostaglandin analogues in glaucoma and docetaxel in prostate cancer has been described. Two plausible hypotheses suggested were either direct stimulation of meibomian gland secretion or meibomian duct inflammation and blockage. ${ }^{11,12} \mathrm{~A}$ viral etiology has also been described as causing chalazia. Herpes, 
Epstein-Barr and adenovirus have all been reported to infect the eyelid sebaceous glands. This provides some indirect evidence to a virus-related etiology for some chalazia. ${ }^{13}$ The authors theorize that perhaps bortezomib facilitates viral infections such as herpes zoster thereby promoting chalazia. ${ }^{14}$

\section{References}

1. Kyle RA, Rajkumar SV. An overview of the progress in the treatment of multiple myeloma. Expert Rev Hematol 2014;7:5-7.

2. Hideshima T, Richardson P, Chauhan D, et al. The proteasome inhibitor PS-341 inhibits growth, induces apoptosis and overcomes drug resistance in human multiple myeloma Cells. Cancer Res 2001;61: 3071-6.

3. Richardson PG, Barlogie B, Berenson J, et al. A phase 2 study of bortezomib in relapsed refractory myeloma. N Engl J Med 2003;348:2609-17.

4. Braunstein MJ, Scott SS, Scott CM, et al. Antimyeloma effects of the heat shock protein 70 molecular chaperone inhibitor MAL3-101. J Oncol 2011;2011:232037.

5. Ghobirial IM, Hong F, Padmanabhan S, et al. Phase II trial of weekly bortezomib in combination with rituximab in relapsed or relapsed and refractory Waldenstrom macroglobulinemia. J Clin Oncol 2010;28: 1422-8.

6. Kanski JJ. Clinical ophthalmology: a systematic approach. 5th ed. 0xford: Butterworth-Heinemann Ltd; 1994.

7. Ballen PH. Inflammations of the lid. Int Ophthalmol Clin 1964;4:5-20.

8. Dhaliwal U, Arora VK, Singh N, et al. Cytopathology of chalazia. Diagn Cytopathol 2004;31:118.

9. Gilchrist H, Lee G. Management of chalazia in general practice. Aust Fam Physician 2009;38:311-4.
10. Grob SR, Jakobiec FA, Rashid A, et al. Chalazia associated with bortezomib therapy for multiple myeloma. Ophthalmology 2014;121:1845-7.

11. Gupta S, Silliman CG, Trump DL. Docetaxel-induced meibomian duct inflammation and blockage leading to chalazion formation. Prostate Cancer Prostatic Dis 2007;10:396-7.

12. Cunniffe MG, Medel-Jimenez R, GonzalezCandial M. Topical antiglaucoma treatment with prostaglandin analogues may precipitate meibomian gland disease. Ophthal Plast Reconstr Surg 2011;27:e128-9.

13. Mansour AM, Chan CC, Crawford MA, et al. Virus-induced chalazion. Eye (Lond.) 2006;20:242-6.

14. Solh M, Fisher RI, Goy A, et al. Herpes zoster complicating bortezomib therapy of relapsed/refractory indolent B-cell and mantle cell lymphoma: an analysis of two phase II trials. Leuk Lymphoma 2013;54: 2185-9. 